Quantifying Impacts of Microcosm Mass Loss on Kinetic Constant Estimation

Supporting

Information

Jack L. Elsey ${ }^{1}$, John A. Christ ${ }^{2}$, and Linda M. Abriola ${ }^{3 *}$

contains a total of:

- 13 pages

- 2 figures

- 3 tables

${ }^{1}$ Tufts University

${ }^{2}$ S\&B Christ Consulting, LLC.

${ }^{3}$ Brown University

* corresponding author: linda_abriola@brown.edu 
2 Experimental measurements and model predictions plotted in the cited papers (Table 1, column 1) were extracted with 3 WebPlotDigitizer ${ }^{1}$ and used (1) to estimate experimental mass losses, if not quantified by the study authors (Table 1, 4 column 4), and (2) to evaluate whether a paper's model fitting method accounted for these mass losses (Table 1, column 5 6). A subset of the studies did not have sufficient information to estimate experimental mass loss. If a study within that 6 subset attempted to model the MRD process it is included in Table 1; other studies ${ }^{2-5}$ were excluded from the table. Two

7 of the studies in Table 1 have an experimental mass loss of " $\approx 0$ " (column 4 ). In one, presented measurements appear to 8 have been corrected for experimental mass loss (Yu et al. ${ }^{6}$ ), and in the other, presented data suggest an increase of mass 9 over time (He et al. ${ }^{7}$ ).

10 All reviewed studies tracked the MRD process over time by monitoring the aqueous concentration of each aliphatic 11 hydrocarbon. Studies that attempted to estimate the total amount of each compound present in the microcosm (e.g. as 12 a plot of moles/bottle versus time ${ }^{8-10}$ ) did so by assuming equilibrium partitioning between the aqueous phase, gaseous 13 headspace, and sorbed soil phase (if present, see column 3 of Table 1). All estimates of experimental mass loss in 14 column 4 of Table 1 were made using this equilibrium partitioning assumption.

15 Measures that may reduce experimental mass loss do not appear to have been widely applied or highly effective. The only studies in this literature review that used Mininert valves to limit mass loss did not fit kinetic MRD models.5,11,12

17 Microcosms with Teflon-lined septa still exhibited mass losses greater than $25 \% .{ }^{13,14}$ Studies that employed lower18 volume headspace sampling regimes (see Table S3) typically lost $20 \%$ or more mass. ${ }^{15,16}$

19 Other information, such as electron donor and experiment duration, are summarized for many of these studies in 20 Chambon et al. ${ }^{17}$ 
22 If it is assumed that partitioning between the aqueous, adsorbed, and gaseous phases is linear and at equilibrium, the

23 left-hand side of Eqn 3 becomes:

$$
\frac{d}{d t}\left(V^{a} C_{i}^{a}\right)+\frac{d}{d t}\left(\frac{V^{g} C_{i}^{a}}{H_{i} R T}\right)+\frac{d}{d t}\left(K_{i}^{d} m_{s} C_{i}^{a}\right)
$$

24 where $H_{i}$ is the Henry's law constant $\left[\mathrm{mmol} / \mathrm{m}^{3} / \mathrm{Pa}\right], \mathrm{R}$ is the gas constant $[\mathrm{J} / \mathrm{K} / \mathrm{mmol}], T$ is temperature $[\mathrm{K}]$, and $K_{i}^{d}$ is the 25 soil adsorption linear partition coefficient [L/g]. Applying the product rule then substituting $\frac{d V^{a}}{d t}=-Q^{a}$ and $\frac{d V^{g}}{d t}=Q^{a}$ 26 yields:

$$
C_{i}^{a}\left(-Q^{a}\right)+V^{a} \frac{d}{d t}\left(C_{i}^{a}\right)+\frac{1}{H_{i} R T}\left[C_{i}^{a}\left(Q^{a}\right)+V^{g} \frac{d}{d t}\left(C_{i}^{a}\right)\right]+K_{i}^{d} m_{s} \frac{d}{d t}\left(C_{i}^{a}\right)
$$

27 Substituting the above expression back into Eqn 3 and rearranging yields Eqn 4 . Note that this equation assumes that the Henry's constant is not a function of time. An assumption consistent with the experimental observations of this study. For experimental systems where there is a pressure increase in the head space due to gas formation, the Henry's constant may need to be adjusted as described below.

31 Derivation of Diffusive Mass Loss Rate Coefficient

32 The mass loss due to diffusive flux of a VOC compound $i$ through a septum is given as

$$
J_{i}=-D_{i}^{r} \frac{d C_{i}^{r}}{d l}
$$


where $D_{i}^{r}$ is the diffusion coefficient for compound $i$ in the septum material phase $r$ and $l$ refers to position along a

34 centerline passing through the septum material blocking the microcosm bottle opening.

35 The rate of diffusive mass loss $J_{i} A^{r}$ through the septum of cross-sectional area $A^{r}$ is first-order dependent on the 36 aqueous phase concentration $C_{i}^{a}$ if the following assumptions hold true:

- The concentration profile of compound $i$ across the septum of thickness $\Delta l$ maintains a steady state such that it decreases linearly from an initial concentration of $C_{i, 0}^{r}$ on the internal face of the septum to a concentration of zero on the external face of the septum.

- The concentration $C_{i, 0}^{r}$ in the septum material phase at the septum/water interface is linearly proportional to the aqueous phase concentration $C_{i}^{a}$. That is,

$$
C_{i, 0}^{r}=K_{i}^{r a} C_{i}^{a}
$$

where $K_{i}^{r a}$ is a constant.

Under the above assumptions, the diffusive mass loss rate becomes

$$
J_{i} A^{r}=D_{i}^{r} \frac{C_{i, 0}^{r}-0}{\Delta l} A^{r}=k_{i}^{d l} C_{i}^{a}
$$

44 where the effective diffusional mass loss rate coefficient $k_{i}^{d l}\left[\mathrm{~L}^{3} \mathrm{~T}^{-1}\right]$ is

$$
k_{i}^{d l}=\frac{A^{r} D_{i}^{r} K_{i}^{r a}}{\Delta l}
$$

45 Note that due to differences in rubber composition and manufacturing techniques, the septa available from different

46 vendors will likely have different diffusive mass loss parameters. Values for $k_{i}^{d l}$ can be estimated using data from short- 
47 term abiotic microcosm experiments (i.e. when $R_{i}^{a}=0$ and total time is less than approximately 50 days). Under no 48 reaction conditions, Eqn 4 becomes:

$$
\frac{d}{d t}\left(C_{i}^{a}\right)=-\frac{\frac{C_{i}^{a} Q^{a}}{H_{i} R T}+k_{i}^{d l} C_{i}^{a}}{V^{a}+\frac{V^{g}}{H_{i} R T}+K_{i}^{d} m_{s}}
$$

49 Solving for $k_{i}^{d l}$ yields:

$$
k_{i}^{d l} \approx\left(\frac{V^{a}}{\overline{C_{i}^{a}}}+\frac{V^{g}}{\overline{C_{i}^{a}} H_{i} R T}+\frac{K_{i}^{d} m_{s}}{\overline{C_{i}^{a}}}\right)\left(-\frac{\Delta C_{i}^{a}}{\Delta t}\right)-\frac{Q^{a}}{H_{i} R T}
$$

50 where $\Delta C_{i}^{a} / \Delta t$ is the slope of the best fit line to the concentration data and $\overline{C_{i}^{a}}$ is the mean of all measured $C_{i}^{a}$ values.

51 Note that if some mass loss mechanism other than sampling or diffusion through the septum (e.g. adsorption to internal

52 surfaces of the microcosm) occurs in the system, its influence on internal concentrations will still be empirically

53 approximated by the $k_{i}^{d l}$ fit to abiotic experiments. In such a scenario this parameter should be deemed an effective

54 mass loss rate coefficient, not a diffusive mass loss rate coefficient.

\section{Microcosm Pressurization}

56 In experimental microcosms where gas formation may occur the Henry's law coefficient $H_{2}$ at system pressure $P_{2}$ can be

57 calculated with $H_{1}$ and $P_{1}$ via the expression

$$
\frac{1}{H_{2}}=\frac{1}{H_{1}} \exp \left[\int_{P_{1}}^{P_{2} \bar{V}^{\infty}} \frac{\bar{R}}{R T}\right]
$$


where $\bar{V}^{\infty}$ is the partial molar volume at infinite dilution, $R$ is the gas constant, and $T$ is temperature..$^{18}$ If it is assumed that $\bar{V}^{\infty}$ is invariant with system pressure ${ }^{19}$ and can be approximated with the pure phase molar volume ${ }^{18}$, then 60 simplifying yields:

$$
\frac{1}{H_{2}} \approx \frac{1}{H_{1}} \exp \left[\frac{V^{m}\left(P_{2}-P_{1}\right)}{R T}\right]
$$

where $V^{m}$ is the pure phase molar volume calculated with the molar weight and tabulated density data ${ }^{20}$. This correction factor is quite small, however, for most aliphatic hydrocarbons under typical experimental conditions.

For example, if the microcosm system described in this paper had an initial lactate concentration of $5 \mathrm{mM}$ and each mole of lactate yielded two moles of carbon dioxide and one mole of methane ${ }^{21}$ application of the ideal gas equation would suggest a pressure increase of less than 0.6 bar. Approximate Henry's law coefficient correction factors (i.e. the exponential in Eqn S10) for such a pressure increase are 1.0025, 1.0022, 1.0019, 1.0017, 1.7979 for PCE, TCE, cis-DCE, VC, and ethene, respectively. These calculations suggest that such a correction would only be needed for $H_{\text {ethene }}$ if the microcosm were extensively pressurized (which was not observed during any of the laboratory experiments that the synthetic data in this paper are based on).

71 It is worth noting, however, that if such a correction were neglected, then even a model with the correct mass loss considerations could still possibly overpredict $C_{\text {ethene }}^{a}$ and underpredict $C_{\text {ethene. Depending on the sampling methodology }}^{g}$

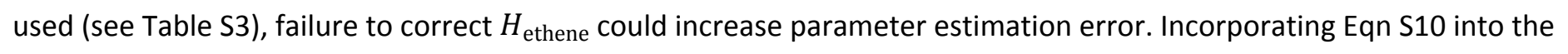
kinetic model presented here (Eqn 4), however, provides a method for more accurately modeling kinetic systems even in the presence of a pressurized microcosm. 


\section{Initial Model Fit}

77 Table 2 provides best-fit model parameter values from the initial model fit, which had a Sum of Squared Residuals (SSR)

78 of $6.54 \mathrm{E}-3 \mathrm{mM}^{2}$ (see Eqn 5). Figure $\mathrm{S} 1$ plots the corresponding modeled and measured aqueous concentrations for this

79 initial scenario. The shapes of the cis-DCE and ethene measurements were well captured by the fitted model (SSR of 8.55E-

804 and $1.08 \mathrm{E}-4 \mathrm{mM}^{2}$, respectively, if the anomalous cis-DCE measurement on day 5 is excluded). Fitted model agreement

81 with the VC measurements was not as good (SSR of $6.82 \mathrm{E}-4 \mathrm{mM}^{2}$ ), but the general timing of the rise and fall of the VC

82 concentration, as well as its peak magnitude were generally consistent with the experimental observations. Figure S1

83 confirms that the fitted model parameters reproduce a realistic TCE to ethene degradation scenario in an experimental 84 microcosm.

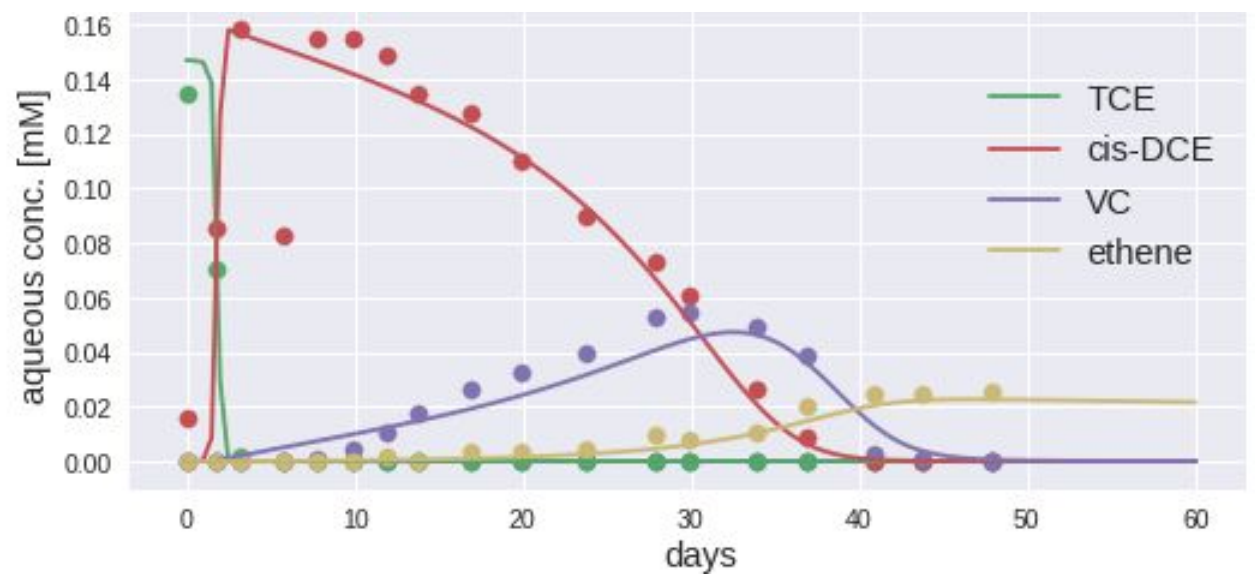




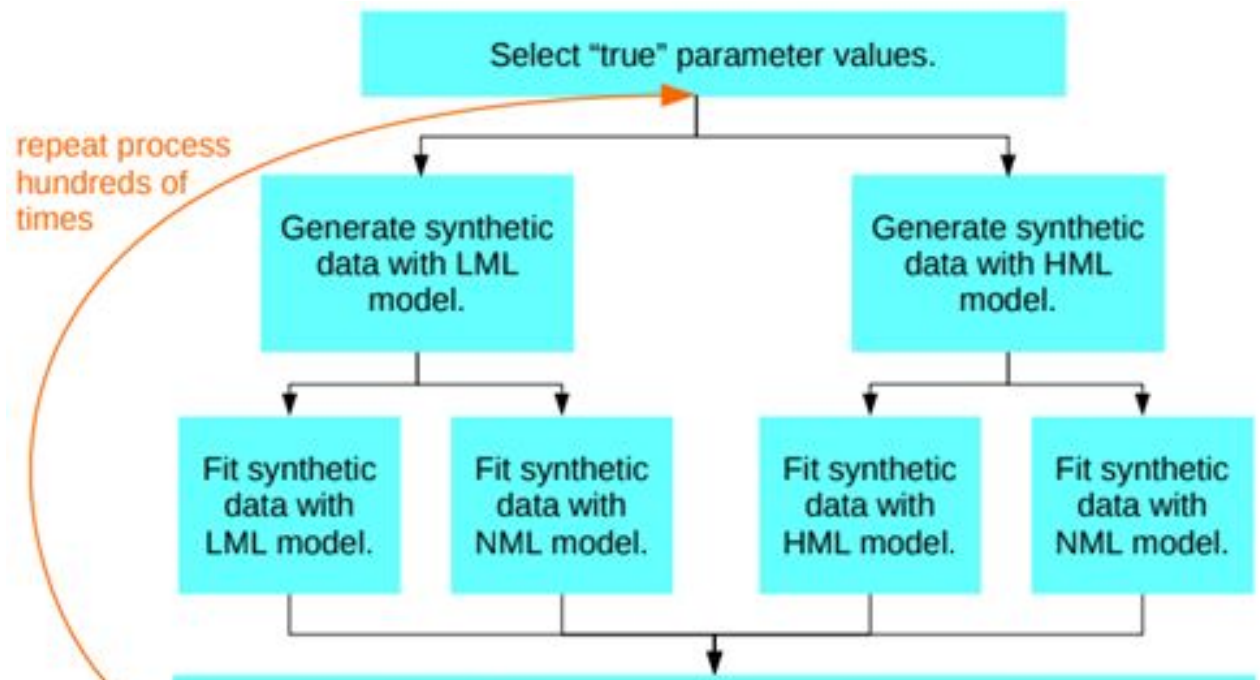

Evaluate fitted parameter bias, using "true" values.

Figure S2: Overall Procedure for Monte Carlo Model Fitting Experiment 
Table S1: Alternative Parameter Values for Additional Model Fitting Experiments (All other parameters match those presented in Table 2.)

\begin{tabular}{|c|c|c|c|c|c|c|c|c|c|c|}
\hline \multirow{2}{*}{ trial } & \multicolumn{2}{|c|}{$\begin{array}{c}\text { maximum substrate utilization rate } \\
{[\mathrm{mmol} /(\mathrm{mg} \text { cell }) / \mathrm{d}]}\end{array}$} & \multicolumn{2}{|c|}{$\begin{array}{l}\text { half saturation coefficients } \\
{[\mathrm{mM}]}\end{array}$} & \multicolumn{2}{|c|}{$\begin{array}{c}\text { aqueous phase sampling rate } \\
{[\mathrm{L} / \text { day }]}\end{array}$} & \multicolumn{4}{|c|}{$\begin{array}{l}\text { HML effective diffusional mass loss rate coefficient } \\
{[\text { [L/day] }}\end{array}$} \\
\hline & $u_{\mathrm{DCE}}^{\max }$ & $u_{V C}^{\max }$ & $K_{\mathrm{DCE}}^{S}$ & $K_{\mathrm{VC}}^{S}$ & $\operatorname{LML} Q^{a}$ & HML $Q^{a}$ & $k_{T C E}^{d l}$ & $k_{D C E}^{d l}$ & $k_{V C}^{d l}$ & $k_{\text {ethene }}^{d l}$ \\
\hline 1 & $2.79 \mathrm{E}-1(\times 0.4)$ & $1.37 \mathrm{E}-1(\times 0.5)$ & $4.44 \mathrm{E}-2(\times 0.50)$ & $8.25 \mathrm{E}-3(\times 0.3)$ & $-1.46 \mathrm{E}-4$ & $-1.33 \mathrm{E}-4$ & $-4.54 \mathrm{E}-4$ & $-5.28 \mathrm{E}-4$ & $-6.58 \mathrm{E}-4$ & $-9.82 \mathrm{E}-4$ \\
\hline 2 & $2.79 \mathrm{E}-1(\times 0.4)$ & $2.74 \mathrm{E}-1(\times 1.0)$ & $4.44 \mathrm{E}-2(\times 0.50)$ & $8.25 \mathrm{E}-3(\times 0.3)$ & $-2.77 \mathrm{E}-4$ & $-2.65 \mathrm{E}-4$ & $-8.38 \mathrm{E}-4$ & $-9.76 \mathrm{E}-4$ & $-1.22 \mathrm{E}-3$ & $-1.81 \mathrm{E}-3$ \\
\hline 3 & $3.49 \mathrm{E}-1(\times 0.5)$ & $1.37 \mathrm{E}-1(\times 0.5)$ & $4.44 \mathrm{E}-2(\times 0.50)$ & $8.25 \mathrm{E}-3(\times 0.3)$ & $-1.75 \mathrm{E}-4$ & $-1.71 \mathrm{E}-4$ & $-5.64 \mathrm{E}-4$ & $-6.57 \mathrm{E}-4$ & $-8.18 \mathrm{E}-4$ & $-1.22 \mathrm{E}-3$ \\
\hline 4 & $3.49 \mathrm{E}-1(\times 0.5)$ & $2.74 \mathrm{E}-1(\times 1.0)$ & $4.44 \mathrm{E}-2(\times 0.50)$ & $8.25 \mathrm{E}-3(\times 0.3)$ & $-3.40 \mathrm{E}-4$ & $-3.27 \mathrm{E}-4$ & $-1.02 \mathrm{E}-3$ & $-1.19 \mathrm{E}-3$ & $-1.48 \mathrm{E}-3$ & $-2.20 \mathrm{E}-3$ \\
\hline 5 & $6.98 \mathrm{E}-1(\times 1.0)$ & $1.10 \mathrm{E}-1(\times 0.4)$ & $4.44 \mathrm{E}-2(\times 0.50)$ & $8.25 \mathrm{E}-3(\times 0.3)$ & $-1.84 \mathrm{E}-4$ & $-1.88 \mathrm{E}-4$ & $-6.77 \mathrm{E}-4$ & $-7.88 \mathrm{E}-4$ & $-9.81 \mathrm{E}-4$ & $-1.46 \mathrm{E}-3$ \\
\hline 6 & $6.98 \mathrm{E}-1(\times 1.0)$ & $2.74 \mathrm{E}-1(\times 1.0)$ & $4.44 \mathrm{E}-2(\times 0.50)$ & $8.25 \mathrm{E}-3(\times 0.3)$ & $-4.86 \mathrm{E}-4$ & $-5.00 \mathrm{E}-4$ & $-1.59 \mathrm{E}-3$ & $-1.85 \mathrm{E}-3$ & $-2.30 \mathrm{E}-3$ & $-3.43 \mathrm{E}-3$ \\
\hline 7 & $2.79 \mathrm{E}-1(\times 0.4)$ & $1.64 \mathrm{E}-1(\times 0.6)$ & $6.66 \mathrm{E}-2(\times 0.75)$ & $8.25 \mathrm{E}-3(\times 0.3)$ & $-1.62 \mathrm{E}-4$ & $-1.37 \mathrm{E}-4$ & $-4.76 \mathrm{E}-4$ & $-5.54 \mathrm{E}-4$ & $-6.90 \mathrm{E}-4$ & $-1.03 \mathrm{E}-3$ \\
\hline 8 & $3.49 \mathrm{E}-1(\times 0.5)$ & $1.37 \mathrm{E}-1(\times 0.5)$ & $6.66 \mathrm{E}-2(\times 0.75)$ & $8.25 \mathrm{E}-3(\times 0.3)$ & $-1.59 \mathrm{E}-4$ & $-1.45 \mathrm{E}-4$ & $-4.95 \mathrm{E}-4$ & $-5.76 \mathrm{E}-4$ & $-7.17 \mathrm{E}-4$ & $-1.07 \mathrm{E}-3$ \\
\hline 9 & $6.98 \mathrm{E}-1(\times 1.0)$ & $1.10 \mathrm{E}-1(\times 0.4)$ & $8.88 \mathrm{E}-2(\times 1.00)$ & $8.25 \mathrm{E}-3(\times 0.3)$ & $-1.64 \mathrm{E}-4$ & $-1.64 \mathrm{E}-4$ & $-5.84 \mathrm{E}-4$ & $-6.80 \mathrm{E}-4$ & $-8.47 \mathrm{E}-4$ & $-1.26 \mathrm{E}-3$ \\
\hline 10 & $4.89 \mathrm{E}-1(\times 0.7)$ & $1.10 \mathrm{E}-1(\times 0.4)$ & $1.11 \mathrm{E}-1(\times 1.25)$ & $8.25 \mathrm{E}-3(\times 0.3)$ & $-1.24 \mathrm{E}-4$ & $-1.03 \mathrm{E}-4$ & $-3.93 \mathrm{E}-4$ & $-4.58 \mathrm{E}-4$ & $-5.70 \mathrm{E}-4$ & $-8.51 \mathrm{E}-4$ \\
\hline 11 & $6.98 \mathrm{E}-1(\times 1.0)$ & $1.10 \mathrm{E}-1(\times 0.4)$ & $1.11 \mathrm{E}-1(\times 1.25)$ & $8.25 \mathrm{E}-3(\times 0.3)$ & $-1.57 \mathrm{E}-4$ & $-1.51 \mathrm{E}-4$ & $-5.43 E-4$ & $-6.32 \mathrm{E}-4$ & $-7.87 \mathrm{E}-4$ & $-1.17 \mathrm{E}-3$ \\
\hline 12 & $3.49 \mathrm{E}-1(\times 0.5)$ & $2.74 \mathrm{E}-1(\times 1.0)$ & $1.33 \mathrm{E}-1(\times 1.50)$ & $8.25 \mathrm{E}-3(\times 0.3)$ & $-2.07 \mathrm{E}-4$ & $-1.62 \mathrm{E}-4$ & $-6.03 E-4$ & $-7.03 E-4$ & $-8.75 \mathrm{E}-4$ & $-1.31 \mathrm{E}-3$ \\
\hline 13 & $2.79 \mathrm{E}-1(\times 0.4)$ & $2.74 \mathrm{E}-1(\times 1.0)$ & $6.66 \mathrm{E}-2(\times 0.75)$ & $1.38 \mathrm{E}-2(\times 0.5)$ & $-2.00 \mathrm{E}-4$ & $-1.71 \mathrm{E}-4$ & $-5.79 \mathrm{E}-4$ & $-6.74 \mathrm{E}-4$ & $-8.39 \mathrm{E}-4$ & $-1.25 \mathrm{E}-3$ \\
\hline 14 & $2.79 \mathrm{E}-1(\times 0.4)$ & $2.74 \mathrm{E}-1(\times 1.0)$ & $8.88 \mathrm{E}-2(\times 1.00)$ & $1.38 \mathrm{E}-2(\times 0.5)$ & $-1.71 \mathrm{E}-4$ & $-1.25 \mathrm{E}-4$ & $-4.73 E-4$ & $-5.51 \mathrm{E}-4$ & $-6.86 \mathrm{E}-4$ & $-1.02 \mathrm{E}-3$ \\
\hline 15 & $6.98 \mathrm{E}-1(\times 1.0)$ & $2.74 \mathrm{E}-1(\times 1.0)$ & $1.33 \mathrm{E}-1(\times 1.50)$ & $1.38 \mathrm{E}-2(\times 0.5)$ & $-3.40 \mathrm{E}-4$ & $-3.21 \mathrm{E}-4$ & $-1.02 \mathrm{E}-3$ & $-1.18 \mathrm{E}-3$ & $-1.47 \mathrm{E}-3$ & $-2.20 \mathrm{E}-3$ \\
\hline 16 & $2.79 \mathrm{E}-1(\times 0.4)$ & $2.74 \mathrm{E}-1(\times 1.0)$ & $4.44 \mathrm{E}-2(\times 0.50)$ & $2.75 \mathrm{E}-2(\times 1.0)$ & $-1.62 \mathrm{E}-4$ & $-1.27 \mathrm{E}-4$ & $-4.52 \mathrm{E}-4$ & $-5.27 \mathrm{E}-4$ & $-6.56 \mathrm{E}-4$ & $-9.79 \mathrm{E}-4$ \\
\hline 17 & $6.98 \mathrm{E}-1(\times 1.0)$ & $1.92 \mathrm{E}-1(\times 0.7)$ & $6.66 \mathrm{E}-2(\times 0.75)$ & $2.75 \mathrm{E}-2(\times 1.0)$ & $-2.20 \mathrm{E}-4$ & $-2.00 \mathrm{E}-4$ & $-7.11 \mathrm{E}-4$ & $-8.27 \mathrm{E}-4$ & $-1.03 E-3$ & $-1.54 \mathrm{E}-3$ \\
\hline 18 & $4.89 \mathrm{E}-1(\times 0.7)$ & $2.74 \mathrm{E}-1(\times 1.0)$ & $4.44 \mathrm{E}-2(\times 0.50)$ & $5.50 \mathrm{E}-2(\times 2.0)$ & $-1.82 \mathrm{E}-4$ & $-1.38 \mathrm{E}-4$ & $-5.30 \mathrm{E}-4$ & $-6.17 E-4$ & $-7.68 \mathrm{E}-4$ & $-1.15 E-3$ \\
\hline 19 & $6.98 \mathrm{E}-1(\times 1.0)$ & $2.74 \mathrm{E}-1(\times 1.0)$ & $4.44 \mathrm{E}-2(\times 0.50)$ & $5.50 \mathrm{E}-2(\times 2.0)$ & $-2.37 \mathrm{E}-4$ & $-2.05 \mathrm{E}-4$ & $-7.48 \mathrm{E}-4$ & $-8.71 \mathrm{E}-4$ & $-1.09 \mathrm{E}-3$ & $-1.62 \mathrm{E}-3$ \\
\hline 20 & $6.98 \mathrm{E}-1(\times 1.0)$ & $2.74 \mathrm{E}-1(\times 1.0)$ & $1.11 \mathrm{E}-1(\times 1.25)$ & $5.50 \mathrm{E}-2(\times 2.0)$ & $-1.84 \mathrm{E}-4$ & $-1.29 \mathrm{E}-4$ & $-5.14 \mathrm{E}-4$ & $-5.98 \mathrm{E}-4$ & $-7.45 \mathrm{E}-4$ & $-1.11 \mathrm{E}-3$ \\
\hline
\end{tabular}

Note: Values given in parentheses indicate a perturbation factor applied to the "true" maximum substrate utilization rate or half saturation coefficient from the Monte Carlo Experiment (see Table 2). 
Table S2: Parameter Estimation Errors for Additional Model Fitting Experiments

\begin{tabular}{|c|c|c|c|c|c|c|c|c|c|c|c|c|c|c|c|c|}
\hline \multirow{2}{*}{ trial } & \multicolumn{4}{|c|}{ HML model fit to HML data } & \multicolumn{4}{|c|}{ NML model fit to HML data } & \multicolumn{4}{|c|}{ LML model fit to LML data } & \multicolumn{4}{|c|}{ NML model fit to LML data } \\
\hline & $u_{\mathrm{DCE}}^{\max }$ & $u_{\mathrm{VC}}^{\max }$ & $K_{\mathrm{DCE}}^{S}$ & $K_{\mathrm{VC}}^{S}$ & $u_{\mathrm{DCE}}^{\max }$ & $u_{V C}^{\max }$ & $K_{\mathrm{DCE}}^{S}$ & $K_{\mathrm{VC}}^{S}$ & $u_{\mathrm{DCE}}^{\max }$ & $u_{V C}^{\max }$ & $K_{\mathrm{DCE}}^{S}$ & $K_{\mathrm{VC}}^{S}$ & $u_{\mathrm{DCE}}^{\max }$ & $u_{\mathrm{VC}}^{\max }$ & $K_{\mathrm{DCE}}^{S}$ & $K_{\mathrm{VC}}^{S}$ \\
\hline 1 & $1 \%$ & $12 \%$ & $0 \%$ & $32 \%$ & $73 \%$ & $-30 \%$ & $341 \%$ & $-88 \%$ & $-4 \%$ & $2 \%$ & $-14 \%$ & $3 \%$ & $-6 \%$ & $-8 \%$ & $-6 \%$ & $28 \%$ \\
\hline 2 & $2 \%$ & $25 \%$ & $1 \%$ & $52 \%$ & $95 \%$ & $-45 \%$ & $341 \%$ & $-88 \%$ & $6 \%$ & $34 \%$ & $11 \%$ & $77 \%$ & $10 \%$ & $10 \%$ & $30 \%$ & $32 \%$ \\
\hline 3 & $-5 \%$ & $-10 \%$ & $-6 \%$ & $-31 \%$ & $74 \%$ & $-25 \%$ & $341 \%$ & $-88 \%$ & $-3 \%$ & $-6 \%$ & $-5 \%$ & $-20 \%$ & $-5 \%$ & $-13 \%$ & $5 \%$ & $-48 \%$ \\
\hline 4 & $-1 \%$ & $1 \%$ & $-3 \%$ & $0 \%$ & $91 \%$ & $-39 \%$ & $341 \%$ & $-88 \%$ & $-3 \%$ & $1 \%$ & $-11 \%$ & $3 \%$ & $-2 \%$ & $-12 \%$ & $2 \%$ & $-26 \%$ \\
\hline 5 & $-5 \%$ & $0 \%$ & $15 \%$ & $6 \%$ & $52 \%$ & $169 \%$ & $107 \%$ & $1109 \%$ & $-3 \%$ & $4 \%$ & $-15 \%$ & $25 \%$ & $7 \%$ & $8 \%$ & $13 \%$ & $60 \%$ \\
\hline 6 & $-4 \%$ & $-9 \%$ & $-6 \%$ & $-29 \%$ & $52 \%$ & $-25 \%$ & $223 \%$ & $-88 \%$ & $-1 \%$ & $-2 \%$ & $-4 \%$ & $-4 \%$ & $-3 \%$ & $-13 \%$ & $12 \%$ & $-46 \%$ \\
\hline 7 & $-3 \%$ & $-7 \%$ & $-3 \%$ & $-19 \%$ & $56 \%$ & $-36 \%$ & $194 \%$ & $-88 \%$ & $-3 \%$ & $-1 \%$ & $-4 \%$ & $-6 \%$ & $-2 \%$ & $-10 \%$ & $8 \%$ & $-28 \%$ \\
\hline 8 & $-1 \%$ & $6 \%$ & $-4 \%$ & $19 \%$ & $51 \%$ & $-28 \%$ & $194 \%$ & $-88 \%$ & $1 \%$ & 79 & $0 \%$ & $22 \%$ & $0 \%$ & $-5 \%$ & $11 \%$ & $-17 \%$ \\
\hline 9 & $-14 \%$ & $-6 \%$ & $-24 \%$ & $-25 \%$ & $32 \%$ & $-14 \%$ & $121 \%$ & $-88 \%$ & $-12 \%$ & $-2 \%$ & $-25 \%$ & $-11 \%$ & $-13 \%$ & $-10 \%$ & $-14 \%$ & $-55 \%$ \\
\hline 10 & $-7 \%$ & $-5 \%$ & $-9 \%$ & $-19 \%$ & $16 \%$ & $-20 \%$ & $77 \%$ & $-88 \%$ & $-8 \%$ & $-5 \%$ & $-10 \%$ & $-23 \%$ & $-7 \%$ & $-11 \%$ & $1 \%$ & $-49 \%$ \\
\hline 11 & $-2 \%$ & $-4 \%$ & $0 \%$ & $-18 \%$ & $19 \%$ & $-15 \%$ & $77 \%$ & $-88 \%$ & $0 \%$ & $-3 \%$ & $4 \%$ & $-16 \%$ & $-1 \%$ & $-12 \%$ & $16 \%$ & $-60 \%$ \\
\hline 12 & $5 \%$ & $11 \%$ & $9 \%$ & $18 \%$ & $-18 \%$ & $56 \%$ & $20 \%$ & $-88 \%$ & $-2 \%$ & $-17 \%$ & $-1 \%$ & $-32 \%$ & $18 \%$ & $-15 \%$ & $37 \%$ & $-24 \%$ \\
\hline 13 & $-10 \%$ & $-42 \%$ & $-13 \%$ & $-71 \%$ & $-23 \%$ & $56 \%$ & $49 \%$ & $-93 \%$ & $-8 \%$ & $-31 \%$ & $-11 \%$ & $-56 \%$ & $-4 \%$ & $-37 \%$ & $6 \%$ & $-64 \%$ \\
\hline 14 & $-10 \%$ & $16 \%$ & $-17 \%$ & $17 \%$ & $41 \%$ & $-58 \%$ & $121 \%$ & $-93 \%$ & $-2 \%$ & $56 \%$ & $-3 \%$ & $85 \%$ & $13 \%$ & $56 \%$ & $30 \%$ & $98 \%$ \\
\hline 15 & $4 \%$ & $-9 \%$ & $9 \%$ & $-18 \%$ & $9 \%$ & $-41 \%$ & $47 \%$ & $-93 \%$ & $2 \%$ & $-10 \%$ & $8 \%$ & $-24 \%$ & $8 \%$ & $-21 \%$ & $26 \%$ & $-47 \%$ \\
\hline 16 & $2 \%$ & $8 \%$ & $5 \%$ & $13 \%$ & $61 \%$ & $-64 \%$ & $327 \%$ & $-96 \%$ & $8 \%$ & $38 \%$ & $18 \%$ & $68 \%$ & $13 \%$ & $9 \%$ & $45 \%$ & $22 \%$ \\
\hline 17 & $-14 \%$ & $-22 \%$ & $-23 \%$ & $-48 \%$ & $36 \%$ & $-42 \%$ & $194 \%$ & $-96 \%$ & $-16 \%$ & $8 \%$ & $-43 \%$ & $17 \%$ & $-9 \%$ & $13 \%$ & $-23 \%$ & $34 \%$ \\
\hline 18 & $0 \%$ & $7 \%$ & $-2 \%$ & $11 \%$ & $49 \%$ & $-64 \%$ & $341 \%$ & $-98 \%$ & $-3 \%$ & $8 \%$ & $-13 \%$ & $15 \%$ & $7 \%$ & $17 \%$ & $20 \%$ & $35 \%$ \\
\hline 19 & $1 \%$ & $-24 \%$ & $9 \%$ & $-40 \%$ & $52 \%$ & $-58 \%$ & $326 \%$ & $-98 \%$ & $8 \%$ & $-5 \%$ & $28 \%$ & $-10 \%$ & $21 \%$ & $19 \%$ & $67 \%$ & $38 \%$ \\
\hline 20 & $-7 \%$ & $-20 \%$ & $-10 \%$ & $-31 \%$ & $2 \%$ & $-63 \%$ & $77 \%$ & $-98 \%$ & $-1 \%$ & $-13 \%$ & $0 \%$ & $-21 \%$ & $16 \%$ & $-11 \%$ & $38 \%$ & $-16 \%$ \\
\hline
\end{tabular}

Notes:

- All errors are relative to and normalized by the alternative "true" parameter values given in Table S1.

- Values highlighted in yellow were pinned to the respective optimization boundary (see Table 2). 
Table S3: Summary of Microcosm Sampling Methodologies

\begin{tabular}{|c|c|c|c|}
\hline Reference Cited & Phase Sampled & Sample Volume $[\mu \mathrm{L}]$ & Microcosm Volume [mL] \\
\hline Smith 23,24 & gaseous & $200-400$ & 2000 \\
\hline Cupples ${ }^{15}$ & gaseous & 250 & 120 \\
\hline Cupples 25 & gaseous & 250 & 120 \\
\hline Cupples $^{26}$ & gaseous & 250 & 120 \\
\hline $\mathrm{Yu}^{6}$ & gaseous & $100-200$ & 156 \\
\hline $\mathrm{He}^{7}$ & gaseous & $100-200$ & 160 \\
\hline Yang, Y. ${ }^{16}$ & gaseous & 250 & 117 \\
\hline $\mathrm{Yu}^{27}$ & gaseous & $100-200$ & 150 \\
\hline $\mathrm{Amos}^{28}$ & aqueous & 1000 & 160 \\
\hline Friis $^{8}$ & aqueous & 1000 & 120 \\
\hline Friis $^{9}$ & aqueous & 1000 & 500 \\
\hline Huang 29 & gaseous & 500 & 160 \\
\hline Schaefer ${ }^{30}$ & gaseous & unknown & $60-7000$ \\
\hline Cichocka $^{13}$ & gaseous & 500 & 120 \\
\hline Haest $^{31}$ & aqueous & 1000 & 120 \\
\hline Sabalowsky 32 & gaseous & 100 & 156 \\
\hline Malaguerra ${ }^{14}$ & aqueous & 1000 & 320 \\
\hline Harkness $^{11}$ & aqueous & $10-1000$ & 250 \\
\hline Bælum ${ }^{10}$ & aqueous & 1000 & 320 \\
\hline Schneidewind $^{33}$ & gaseous & unknown & 160 \\
\hline Yang, L. ${ }^{22}$ & aqueous & 1000 & 160 \\
\hline
\end{tabular}




\section{References}

(1) Rohatgi, A. WebPlotDigitizer; San Francisco, California, USA, 2019.

(2) Duhamel, M.; Wehr, S. D.; Yu, L.; Rizvi, H.; Seepersad, D.; Dworatzek, S.; Cox, E. E.; Edwards, E. A. Comparison of Anaerobic Dechlorinating Enrichment Cultures Maintained on Tetrachloroethene, Trichloroethene, Cis-Dichloroethene and Vinyl Chloride. Water Research 2002, 36 (17), 4193-4202. https://doi.org/10.1016/S0043-1354(02)00151-3.

(3) Holmes, V. F.; He, J.; Lee, P. K. H.; Alvarez-Cohen, L. Discrimination of Multiple Dehalococcoides Strains in a Trichloroethene Enrichment by Quantification of Their Reductive Dehalogenase Genes. Appl. Environ. Microbiol. 2006, 72 (9), 5877-5883. https://doi.org/10.1128/AEM.00516-06.

(4) Bunge, M.; Kleikemper, J.; Miniaci, C.; Duc, L.; Muusse, M. G.; Hause, G.; Zeyer, J. Benzoate-Driven Dehalogenation of Chlorinated Ethenes in Microbial Cultures from a Contaminated Aquifer. Appl Microbiol Biotechnol 2007, 76 (6), 1447-1456. https://doi.org/10.1007/s00253-007-1097-3.

(5) Duhamel, M.; Edwards, E. A. Growth and Yields of Dechlorinators, Acetogens, and Methanogens during Reductive Dechlorination of Chlorinated Ethenes and Dihaloelimination of 1,2-Dichloroethane. Environ. Sci. Technol. 2007, 41 (7), 2303-2310. https://doi.org/10.1021/es062010r.

(6) Yu, S.; Semprini, L. Kinetics and Modeling of Reductive Dechlorination at High PCE and TCE Concentrations. Biotechnology and Bioengineering 2004, 88 (4), 451-464. https://doi.org/10.1002/bit.20260.

(7) He, J.; Sung, Y.; Krajmalnik-Brown, R.; Ritalahti, K. M.; Löffler, F. E. Isolation and Characterization of Dehalococcoides Sp. Strain FL2, a Trichloroethene (TCE)- and 1,2Dichloroethene-Respiring Anaerobe. Environ. Microbiol. 2005, 7 (9), 1442-1450. https://doi.org/10.1111/j.1462-2920.2005.00830.x.

(8) Friis, A. K.; Heimann, A. C.; Jakobsen, R.; Albrechtsen, H.-J.; Cox, E.; Bjerg, P. L. Temperature Dependence of Anaerobic TCE-Dechlorination in a Highly Enriched Dehalococcoides-Containing Culture. Water Research 2007, 41 (2), 355-364. https://doi.org/10.1016/j.watres.2006.09.026.

(9) Friis, A. K.; Kofoed, J. L. L.; Heron, G.; Albrechtsen, H.-J.; Bjerg, P. L. Microcosm Evaluation of Bioaugmentation after Field-Scale Thermal Treatment of a TCEContaminated Aquifer. Biodegradation 2007, 18 (6), 661-674. https://doi.org/10.1007/s10532-006-9098-y.

(10) Bælum, J.; Scheutz, C.; Chambon, J. C.; Jensen, C. M.; Brochmann, R. P.; Dennis, P.; Laier, T.; Broholm, M. M.; Bjerg, P. L.; Binning, P. J.; Jacobsen, C. S. The Impact of Bioaugmentation on Dechlorination Kinetics and on Microbial Dechlorinating Communities in Subsurface Clay Till. Environmental Pollution 2014, 186, 149-157. https://doi.org/10.1016/j.envpol.2013.11.013. 
(11) Harkness, M.; Fisher, A.; Lee, M. D.; Mack, E. E.; Payne, J. A.; Dworatzek, S.; Roberts, J.; Acheson, C.; Herrmann, R.; Possolo, A. Use of Statistical Tools to Evaluate the Reductive Dechlorination of High Levels of TCE in Microcosm Studies. Journal of Contaminant Hydrology 2012, 131 (1), 100-118. https://doi.org/10.1016/j.jconhyd.2012.01.011.

(12) Duhamel, M.; Edwards, E. A. Microbial Composition of Chlorinated Ethene-Degrading Cultures Dominated by Dehalococcoides. FEMS Microbiol Ecol 2006, 58 (3), 538-549. https://doi.org/10.1111/j.1574-6941.2006.00191.x.

(13) Cichocka, D.; Nikolausz, M.; Haest, P. J.; Nijenhuis, I. Tetrachloroethene Conversion to Ethene by a Dehalococcoides-Containing Enrichment Culture from Bitterfeld. FEMS Microbiol Ecol 2010, 72 (2), 297-310. https://doi.org/10.1111/j.1574-6941.2010.00845.x.

(14) Malaguerra, F.; Chambon, J. C.; Bjerg, P. L.; Scheutz, C.; Binning, P. J. Development and Sensitivity Analysis of a Fully Kinetic Model of Sequential Reductive Dechlorination in Groundwater. Environ. Sci. Technol. 2011, 45 (19), 8395-8402. https://doi.org/10.1021/es201270z.

(15) Cupples, A. M.; Spormann, A. M.; McCarty, P. L. Growth of a Dehalococcoides-Like Microorganism on Vinyl Chloride and Cis-Dichloroethene as Electron Acceptors as Determined by Competitive PCR. Appl. Environ. Microbiol. 2003, 69 (2), 953-959. https://doi.org/10.1128/AEM.69.2.953-959.2003.

(16) Yang, Y.; Pesaro, M.; Sigler, W.; Zeyer, J. Identification of Microorganisms Involved in Reductive Dehalogenation of Chlorinated Ethenes in an Anaerobic Microbial Community. Water Research 2005, 39 (16), 3954-3966. https://doi.org/10.1016/j.watres.2005.07.010.

(17) Chambon, J. C.; Bjerg, P. L.; Scheutz, C.; Bælum, J.; Jakobsen, R.; Binning, P. J. Review of Reactive Kinetic Models Describing Reductive Dechlorination of Chlorinated Ethenes in Soil and Groundwater. Biotechnol. Bioeng. 2013, 110 (1), 1-23. https://doi.org/10.1002/bit.24714.

(18) Koretsky, M. D. Engineering and Chemical Thermodynamics; Wiley, 2004.

(19) Carroll, J. J. What Is Henry’s Law? Chemical Engineering Progress 1991, 87 (9), 48-52.

(20) Haynes, W. M. CRC Handbook of Chemistry and Physics, 96th Edition; CRC Press, 2015.

(21) Thauer, R. K.; Jungermann, K.; Decker, K. Energy Conservation in Chemotrophic Anaerobic Bacteria. Bacteriological reviews 1977, 41 (1), 100-180.

(22) Yang, L.; Jason P. Hnatko; Jack L. Elsey; John A. Christ; Kurt D. Pennell; Natalie L. Cápiro; Linda M. Abriola. Exploration of Processes Governing Microbial Reductive Dechlorination in a Heterogeneous Aquifer Flow Cell. Water Research 2021, 193. https://doi.org/10.1016/j.watres.2021.116842.

(23) Smith, L. H.; Kitanidis, P. K.; McCarty, P. L. Numerical Modeling and Uncertainties in Rate Coefficients for Methane Utilization and TCE Cometabolism by a Methane-Oxidizing Mixed Culture. Biotechnol. Bioeng. 1997, 53 (3), 320-331. https://doi.org/10.1002/(SICI)1097-0290(19970205)53:3<320::AID-BIT11>3.0.CO;2-O. 
(24) Smith, L. H.; McCarty, P. L.; Kitanidis, P. K. Spreadsheet Method for Evaluation of Biochemical Reaction Rate Coefficients and Their Uncertainties by Weighted Nonlinear Least-Squares Analysis of the Integrated Monod Equation. Appl. Environ. Microbiol. 1998, 64 (6), 2044-2050.

(25) Cupples, A. M.; Spormann, A. M.; McCarty, P. L. Comparative Evaluation of Chloroethene Dechlorination to Ethene by Dehalococcoides-like Microorganisms. Environ. Sci. Technol. 2004, 38 (18), 4768-4774. https://doi.org/10.1021/es049965z.

(26) Cupples, A. M.; Spormann, A. M.; McCarty, P. L. Vinyl Chloride and Cis-Dichloroethene Dechlorination Kinetics and Microorganism Growth under Substrate Limiting Conditions. Environ. Sci. Technol. 2004, 38 (4), 1102-1107. https://doi.org/10.1021/es0348647.

(27) Yu, S.; Dolan, M. E.; Semprini, L. Kinetics and Inhibition of Reductive Dechlorination of Chlorinated Ethylenes by Two Different Mixed Cultures. Environ. Sci. Technol. 2005, 39 (1), 195-205. https://doi.org/10.1021/es0496773.

(28) Amos, B. K.; Christ, J. A.; Abriola, L. M.; Pennell, K. D.; Löffler, F. E. Experimental Evaluation and Mathematical Modeling of Microbially Enhanced Tetrachloroethene (PCE) Dissolution. Environ. Sci. Technol. 2007, 41 (3), 963-970. https://doi.org/10.1021/es061438n.

(29) Huang, D.; Becker, J. G. Determination of Intrinsic Monod Kinetic Parameters for Two Heterotrophic Tetrachloroethene (PCE)-Respiring Strains and Insight into Their Application. Biotechnology and Bioengineering 2009, 104 (2), 301-311. https://doi.org/10.1002/bit.22421.

(30) Schaefer, C. E.; Condee, C. W.; Vainberg, S.; Steffan, R. J. Bioaugmentation for Chlorinated Ethenes Using Dehalococcoides Sp.: Comparison between Batch and Column Experiments. Chemosphere 2009, 75 (2), 141-148. https://doi.org/10.1016/j.chemosphere.2008.12.041.

(31) Haest, P. J.; Springael, D.; Smolders, E. Dechlorination Kinetics of TCE at Toxic TCE Concentrations: Assessment of Different Models. Water Research 2010, 44 (1), 331-339. https://doi.org/10.1016/j.watres.2009.09.033.

(32) Sabalowsky, A. R.; Semprini, L. Trichloroethene and Cis-1,2-Dichloroethene Concentration-Dependent Toxicity Model Simulates Anaerobic Dechlorination at High Concentrations: I. Batch-Fed Reactors. Biotechnology and Bioengineering 2010, 107 (3), 529-539. https://doi.org/10.1002/bit.22776.

(33) Schneidewind, U.; Haest, P. J.; Atashgahi, S.; Maphosa, F.; Hamonts, K.; Maesen, M.; Calderer, M.; Seuntjens, P.; Smidt, H.; Springael, D.; Dejonghe, W. Kinetics of Dechlorination by Dehalococcoides Mccartyi Using Different Carbon Sources. Journal of Contaminant Hydrology 2014, 157, 25-36. https://doi.org/10.1016/j.jconhyd.2013.10.006. 